Let us now classify the symptoms which the above cases presented, and see how far they may assist us in establishing a diagnosis.

First, with regard to intelligence.

There was a peculiar obtuseness of intellect, characterised chiefly by apathy to external objects, and a great tendency to drowsiness. There also was evinced a peculiar irritability of temper.

Andral states, that in adults, headache, with severe exacerbations, is a prominent symptom; this also occurred in the last case detailed by me. He also men. tions weakness of the limbs, often ending in paralysis, convulsion, epilepsy; but these symptoms belong to the second period of this state, when it has passed into the acute stage; but as it is an essential feature of every hypertrophy to develop itself slowly, $I$ have only had an opportunity of studying this state in the first or chronic period, as the two first children died of other diseases before they had reached the second period, which most probably would occur about the second dentition; thus in observation 4th of Andral, the boy became epileptic at the age of seven years, and died comatose, after a fit, at nine years old.

The appetite was very great in all the cases which I have noted, and there existed the peculiar projection of the parietal protuberances, on which Dr. Munchmeyer particularly insists, and which, I think, may prove a valuable guide in aiding to discriminate this state from chronic hydrocephalus, with which disease it is most frequently confounded. Thus, in the first case related by Dr. Sims, the mother of the child informed him that they had wished to tap the head at one of the hospitals. And Dr. Hennis Green,* in an excellent article on this subject, states that he recently had seen a child who had been condemned to death by a medical man, as having water on the brain, but which was a case of simple hypertrophy, and which did not interfere with the health of the child. The diagnostic sign which he gives, is the sensation of firmness communicated to the finger, on pressure being made over the fontanelles, in cases of hypertrophy, as contrasted with the fluctuating feel in cases of chronic hydrocephalus; but this could not apply in cases of very young children, or in extreme cases.

The prognosis in children is not necessarily unfavorable, for as it is rather an error of development than an actual disease, there is a natural tendency to return to the normal state; the chief danger, in fact, arising from the occurrence of other diseases, as those attendant on dentition, the exanthemata. It is probable that the rapid and sudden deaths which occasionally take place in the malignant forms of scarlatina (some of which I have myself witnessed when the throat has been but slightly affected, and when they die in a very early stage with cerebral symptoms, as if poisoned by the virulence of the disease), may be the result of a state of hyperæmia, superadded to this peculiar state of hypertrophy of the brain. I merely hint at this, from having found myself quite unable to explain the cause of death by the post mortem appearances in some of those cases which I have unfortunately met with, and which appear to set all treatment at defiance. The causes of this state are very obscure, but are probably dependant on, or connected with struma; and although the observations - Provincial Medical and Surgical Journal. which have been hitherto published with regard to this state, bear reference to it as chiefly occurring in adult life, yet, I think, we must regard it as the result either of an abnormal development of the brain, excited before birth, or depending on primary organisation. For as Tiedeman and Valentine have established that the fœtal brain is one of the heaviest and most vascular organs of the body, but presenting little trace of organisation, the primitive type may continue for some years, and thus give rise to hypertrophy, which may remain for a considerable time without interfering with the general health (as we see occurring in other organs, the liver for instance), unless there be either an increase of intensity in its action, or some acute disease supervenes. Thus bearing out the general law of hypertrophy, when existing in other organs, and which is its peculiar featuro-viz., that the functional disorders which it entails only extend beyond the affected part, according as the part itself extends its sphere of action, thus differing from other oyganic lesions which so soon affect the whole economy, wherever may be the seat of the disease.-Dublin Journal, September, 1842.

\section{THE THEORY OF RESPIRATION.}

\section{By Justus Liebig, M.D.}

During the passage of the venous blood through the lungs, the globules change their color; and with this change of color, oxygen is absorbed from the atmosphere. Further, for every volume of oxygen absorbed, an equal volume of carbonic acid is, in most cases, given out.

The red globules contain a compound of iron; and no other constituent of the body contains iron.

Whatever change the other constituents of the blood undexgo in the lungs, thus much is certain, that the globules of venous blood experience a change of color, and that this change depends on the action of oxygen.

Now we observe that the globules of arterial blood retain their color in the larger vessels, and lose it only during their passage through the capillaries. All those constituents of venous blood, which are capable of combining with oxygen, take up a corresponding quantity of it in the lungs. Experiments made with arterial serum have shown, that when in contact with oxygen it does not diminish the volume of that gas. Venous blood, in contact with oxygen, is reddened, while oxygen is absorbed ; and a corresponding quantity of carbonic acid is formed.

It is evident that the change of color in the venous globules depends on the combination of some one of their elements with oxygen; and that this absorption of oxigen is attended with the separation of a certain quantity of carbonic acid gas.

This carbonic acid is not separated from the serum; for the serum does not possess the property, when in contact with oxygen, of giving off carbonic acid. On the contrary, when separated from the globules, it absorbs from half its volume to an equal volume of carbonic acid, and, at ordinary temperatures, is not saturated with that gas. (See the article "Blut" in the "Handwörterbuch der Chemie von Poggendorff, Wöhler, and Liebig, p. 877.) 
Arterial blood, when drawn from the body, is soon altered; its florid color becomes dark red. The florid blood, which owes its color to the globules, becomes dark by the action of carbonic acid, and this change of color affects the globules, for florid blood absorbs a number of gases which do not dissolve in the fluid part of the blood when separated from the globules. It is evident, therefore, that the globules have the power of combining with gases.

The globules of the blood change their color in different gases; and this change may be owing either to a combination or to a decomposition.

Sulphuretted hydrogen turns them blackish green and finally black; and the original red color cannot, in this case, be restored by contact with oxygen. Here a decomposition has obviously taken place.

The globules darkened by carbonic acid become again florid in oxygen, with disengagement of carbonic acid. The same thing takes place in nitrous oxide. It is clear that they have here undergone no decomposition, and, consequently, they possess the power of combining with gases, while the compound they form with carbonic acid is destroyed by oxygen. When left to themselves, out of the body, the compound formed with oxygen again becomes dark, but does not recover its florid color a second time by the -action of oxygen.

The globules of the blood contain a compound of iron. From the never-failing presence of iron in red blood, we must conclude, that it is unquestionably necessary to animal life; and, since physiology has proved, that the globules take no share in the process of nutrition, it cannot be doubted that they play a part in the process of respiration.

The compound of iron in the globules has the characters of an oxidised compound; for it is decomposed by sulphuretted hydrogen, exactly in the same way as the oxides or other analogous compounds of iron. By means of diluted mineral acids, peroxide (sesquioxide) of iron may be extracted, at the ordinary temperature, from the fresh or dried red coloring matter of the blood.

The characters of the compounds of iron may, perhaps, assist us to explain the share which that metal takes in the respiratory process. No other metal can be compared with iron, for the remarkable properties of its compounds.

The compounds of protoxide of iron possess the property of depriving other oxidised compounds of oxygen; while the compounds of peroxide of iron, under other circumstances, give up oxygen with the utmost facility.

Hydrated peroxide of iron, in contact with organic matters destitute of sulphur, is converted into carbonate of the protoxide.

Carbonate of protoxide of iron, in contact with water and oxygen, is decomposed; all the carbonic acid is given off, and, by absorption of oxygen, it passes into the hydrated peroxide, which may again be converted into a compound of the protoxide.

Not only the oxides of iron, but also the cyanides of that metal, exhibit similar properties. Prussian blue contains iron in combination with all the organic elements of the body; hydrogen and oxygen (water), carbon and nitrogen (cyanogen).

When it is exposed to light, cyanogen is given off, and it becomes white; in the dark it attracts oxygen, and recovers its blue color.

All these observations, taken together, lead to the opinion that the globules of arterial blood contain a compound of iron saturated with oxygen, which, in the living blood, loses its oxygen during its passage through the capillaries. The same thing occurs when it is separated from the body, and begins to undergo decomposition (to putrefy). The compound, rich in oxygen, passes, therefore, by the loss of oxygen (reduction), into one far less charged with that element. One of the products of oxidation formed in this process is carbonic acid. The compound of iron in the venous blood possesses the property of combining with carbonic acid; and it is obvious that the globules of the arterial blood, after losing a part of their oxygen, will, if they meet with carbonic acid, combine with that substance.

When they reach the lungs, they will again take up the oxygen they have lost; for every volume of oxygen absorbed, a corresponding volume of carbonic acid will be separated; they will return to their former state-that is, they will again acquire the power of giving off oxygen.

For every volume of oxygen which the globules can give off, there will be formed (as carbonic acid contains its own volume of oxygen, without condensation) neither more nor less than an equal volume of carbonic acid. For every volume of oxygen which the globules are capable of absorbing, no more carbonic acid can possibly be separated than that volume of oxygen can produce.

When carbonate of protoxide of iron, by the absorption of oxygen, passes into the hydrated peroxide, there are given off, for every volume of oxygen necessary to the change from protoxide to peroxide, four volumes of carbonic acid gas.

But from one volume of oxygen only one volume of carbcnic acid can be produced; and the absorption of one volume of oxygen can only cause, directly, the separation of an equal volume of carbonic acid. Consequently, the substance or compound which has lost its oxygen, during the passage of arterial into venous blood, must have been capable of absorbing or combining with carbonic acid; and we find, in point of fact, that the living blood is never, in any state, saturated with carbonic acid; that it is capable of taking up an additional quantity, without any apparent disturbance of the function of the globules. Thus, for example, after drinking effervescing wines, beer, or mineral waters, more carbonic acid must necessarily be expired than at other times. In all cases, where the oxygen of the arterial globutes has been partly expended, otherwise than in the formation of carbonic acid, the amount 'of this latter gas expired will correspond exactly with that which has been formed; less, however, will be given out after the use of fat and of still wines, than after champagne.

According to the views now developed, the globules of arterial blood, in their passage through the capillaries, yield oxygen to certain constituents of the body. A small portion of this oxygen serves to produce the change of matter, and determines the separation of living parts and their conversion into lifeless compounds, as well as the formation of the secretions and excretions. The greater part, however, of the oxyg en 
is employed in converting into oxidised compounds the newly-formed substances, which no longer form part of the living tissues.

In their return towards the heart, the globules which have lost their oxygen combine with carbonic acid, producing renous blood; and, when they reach the lungs, an exchange takes place between this carbonic acid and the oxygen of the atmosphere.

The organic compound of iron, which exists in venous blood, recovers in the lungs the oxygen it has lost, and, in consequence of this absorption of oxygen, the carbonic acid in combination with it is separated.

All the compounds present in venous blood, which have an attraction for oxygen, are converted, in the lungs, like the globules, into more highly oxidised compounds; a certain amount of carbonic acid is formed, of which a part always remains dissolved in the serum of the blood.

The quantity of carbonic acid dissolved, or of that combined with soda, must be equal in renous and arterial blood, since both have the same temperature; but arterial blood, when drawn, must, after a short time, contain a larger quantity of sarbonic acid than venous blood, because the oxygen of the globules is expended in produci ' $g$ that compound.

Hence, in the animal organism, two processes of oxidation are going on; one in the lungs, the other in the capillaries. By means of the former, in spite of ths degree of cooling, and of the increased evaporation which takes place there, the constant temperature of the lungs is kept up; while the heat of the rest of the body is supplied by the latter.

A man, who expires daily $13.9 \mathrm{oz}$. of carbon, in the form of carbonic acid, consumes, in 24 hours, $37 \mathrm{oz}$. of oxygen, which occupy a space equal to 807 litres $=51,648$ cubic inches (Hessian).

If we reckon 18 respirations to a minute, we have, in 24 hours, 25,920 respirations; and, consequently, in each respiration, there are taken into the blood $\frac{51648}{25} \frac{64}{2}=1.99$ cubic inch of oxygen.

In one minute, therefore, there are added to the constituents of the blood $\times 181.99=35.8$ cubic inches of oxygen, which, at the ordinary temperature, weigh rather less that 12 grains.

If we now assume, that in one minute $10 \mathrm{lbs}$. of blood pass through the lungs (Müller, Physiologie, vol. i, p. 345), and that this quantity of blood measures 320 cubic inches, then 1 cubic inch of oxygen unites with 9 cubic inches of blood, very nearly.

According to the researches of Dinis, Richardson, and Nasse (Handwörterbuch der Physiologie, vol. i. p. 138), 10,000 parts of blood contain 8 parts of peroxide of iron. Consequently, 76,800 grains (10 lbs. Hessian) of blood contain 61.54 grains of peroxide of iron in arterial blood, $=55.14$ of protoxide in venous blood.

Let us now assume that the iron of the globules of venous blood is in the state of protoxide. It follows, that 55.14 grains of protoxide of iron, in passing through the lungs, take up, in one minute, 6.40 grains of oxygen (the quantity necessary to convert it into peroxide). But since, in the same time, the $10 \mathrm{lbs}$. of blood have taken up 12 grains of oxygen, there remain 5.60 grains of oxygen, which combine with the other constituents of the blood.
Now, 55.14 grains of protoxide of iron combine with 34.8 grains of carbonic acid, which occupy the volume of 73 cubic inches. It is, obvious, therefore, that the amount of iron present in the blood, if in the state of protoxide, is sufficient to furnish the means of carrying or transporting twice as much carbonic acid as can possibly be formed by the oxygen absorbed in the lungs.

The hypothesis just developed rests on well-known observations, and, indeed, explains completely the process of respiration, as far as it depends on the globules of the blood. It does not exclude the opinion that carbonic acid may reach the lungs in other ways; that certain other constituents of the blood may give rise to the formation of carbonic acid in the lungs. But all this has no connection with that vital process by which the heat necessary for the support of life is generated in every part of the body. Now it is this alone which, for the present, can be considered as the object truly worthy of investigation. It is not, indeed, uninteresting to inquire, why dark blood becomes florid by the action of nitre, common salt, \&c.; but this question has no relation to the natural respiratory process.

The frightful effects of sulphuretted hydrogen, and of prussic acid, which, when inspired, put a stop to all the phenomena of motion in a few seconds, are explained in a natural manner by the well-known action of these compounds on those of iron, when alkalies are present; and free alkali is never absent in the blood.

Let us suppose that the globules lose their property of absorbing oxygen, and of afterwards giving up this oxygen and carrying off the resulting carbonic acid; such a hypothetical state of disease must instantly become perceptible in the temperature and other vital phenomena of the body. The change of matter will be arrested, while yet the vital motions will not be instantly stopped.

The conductors of force, the nerves, will convey, as before, to the heart and intestines the power necessary for their functions. This power they will receive from the muscular system, while, as no change of matter takes place in the latter, the supply must soon fail. As no change of matter occurs, no lifeless compounds are separated, neither bile nor urine can be formed; and the temperature of the body must sink.

This state of matters soon puts a stop to the process of nutrition, and, sooner or later, death must follow, but unaccompanied by febrile symptoms, which, in this case, is a very important fact.

This example has been selected in order to show the importance and probable advantage of an examination of the blood in analogous diseased conditions. It cannot be, in the slightest degree, doubtful that the function ascribed to the blood globules may be considered as fully explained and cleared up, if in such morbid conditions, we shall discover a change in their form, structure, or chemical charactes-a change which must be recognisable by the use of appropriate reagents.

If we consider the force which determines the vital phenomena as a property of certain substances, this view leads of itself to a new and more rigorous consideration of certain singular phenomena, which these 
very substances exhibit, in circumstances in which they no longer make a part of living organisms.-From Liebig's Animal Chemistry.

\section{PROVINCIAL MEDICAL JOURNAL}

SATURDAY, SEPTEMBER 17, 1842.

On the 2nd of September, at two o'clock, an inquest was held at the Elephant and Castle, Camden Town, on the bodies of two of four boys who had been exposed during several hours of the night to the gases escaping from a lime-kiln in that neighbourhood. This inquiry, and the melancholy accident itself, we shall briefly notice.

The four boys had been found lying in a group upon the kiln, near the opening through which the gases escaped, "the kiln had been left burning over night," and there was every reason for supposing the boys had not approached it until a pretty late hour on the preceding evening. Carrol and Burke (to use the words of the "Times") "were quite dead, both apparently from suffocation; the latter also was much burnt about the arms and legs." The others lay in a state of coma, occasionally interrupted by convulsions, and were removed to University College Hospital, where one shortly afterwards died. We shall at present confine ourselves to the cases of Carrol and Burke, as the report of the inquest on the body of him who died in hospital has not as yet reached us.

It appears, from the account which the "Times" gives of the accident and inquest, that the bodies of Burke and Carrol, being found deprived of circulation and respiration, were looked upon as quite beyond recovery, and no effort whatever was made to restore them to life. Now this was unquestionably a most unfortunate blunder, one which shows clearly, how dangerous it is that a practitioner should take the responsibility of cases, of such rare occurrence in this country, without availing himself of the advice of some gentleman, whose experience and knowledge of medical jurisprudence render it impossible that such an unlucky omission could occur.

It is well known to persons skilled in legal medicine that, under proper treatment, persons recover from the deleterious effects of carbonic acid after many hours of exposure to the gas, and even after many hours' suspension of the circulation and respitatory movements, and it is laid down as a principle, that we should never despair of restoring animation until cadaveric rigidiky is unequivocally established. If the limbs continue flexible, respiration should be imitated unceasingly for several hours (or until the occurrence of rigidity), by pressurt made about twenty times in the minute upon the abdomen in the direction of the diaphragm, and simultaneously on the chest in the direction of the spine; these compressions should be assisted by the process called by the French, aspiration," which consists in drawing air from the lungs through a nostril-tube, and may be executed by the mouth of an assistant or by Weiss' stomach pump; thus a certain quantity of air will be withdrawn from the cavity of the chest about sixteen times in the minute, and an equal quantity of fresh atmospheric air drawn in when the capacity of the thorax is restored by natural elasticity. + At the same time constant efforts should be made to stimulate the respiratory muscles, by galvanism, by the occasional dashing against the body of cold water if its temperature be natural, hot water (at $100^{\circ}$ or $\left.102^{\circ}\right)$ if the temperature of the body be reduced, by careful drying and diligent friction after each affusion, and finally by flagellation, which proved so advantageous lately in a case of asphyxia from drowning reported by Dr. Ogier Ward, of Wolverhampton. These various means may be employed in succession if circumstances permit; if not, any one or two should be patiently continued. Unquestionably the great object is to excite the action of the respiratory muscles; respiration will always prove the best means of removing sanguineous congestion; but it has been proved by experiment on animals, that when the action of the heart is suspended and cannot be excited by the ordinary stimulants, the action of these stimulants will produce contractions of the heart immediately after the abstraction of a very small quantity of blood from the veins connected with the right side of that organ. Hence, if the measures tried during the first half hour be ineffectual, three or four ounces of blood may be taken from the jugular vein. This may be done at once if the asphyxia exists in a strong, well-fed individual. During the administration of the remedial means the body should be laid on a table in a room which, in cold weather, should be well warmed. The patient should be carefully removed to a warm bed when the respiratory muscles are beginning to act well. We have not recommended artificial respiration by means of the bellows and nostril-tube, because the apparatus is not always at hand, and its use is dangerous, even in the hands of medical men; indeed the injection of air into the lungs by a bellows is a very different thing from the ratural entrance of atr into an increased

- During the process it may be prudent to press the larymx moderately against the cesophagus, provided a medical man be present. During the drawing out of the piston rod the mouth and the other nostril should be accurately closed.

+ The Humane Society at present employs Mr. Reid's pump, the price of which is nine guineas; it is an excellent instrument for those who advocate the injection of air, and is constructed so that it accomplishes aspiration also. Mr. Weis will sell for thirty-two shillings a pump of twice the capacity of his stomach pump; the increase of size, although not indispensable, would be adrantageous. The piston of Weiss' pump should be forced home whilst the index is opposite the letter $S$, and drawn out whilst the index lies over the letter $B$; the tube should be attached at orifice $B$. 